\title{
Barriers to Prompt Malaria Diagnosis and Effective Treatment among Children Under Five Years of Age in Mpika District
}

\author{
Article by David Silweya \\ Ph.D. in Public Health, Texila American University, Zambia \\ E-mail: silweyadavid@yahoo.com
}

\begin{abstract}
Background: Prompt malaria diagnosis and effective treatment, is a key malaria control strategy which reduces morbidity and mortality in many settings. However, in Zambia studies show that, only 19\% caretaker of children under five years of age accessed prompt and effective treatment in 2010 at health facilities. The factors contributing to this problem are not well understood in Zambia. The objective of this study is to determine barriers to prompt diagnosis and effective malaria treatment among children under the age of five years in Mpika district.

Methods: An analytical cross section study was conducted in Mpika district of Zambia using both the quantitative and qualitative methods with sample size was 380 caregivers and 8 Focused Group Discussions (FGDs) respectively.

Results: The study found that only $13.9 \%$ of children diagnosed with malaria received prompt malaria treatment. The following variables were found to significantly predict uptake of prompt malaria treatment; distance of less than five kilometers to the health facility (Adjusted OR 2.45 95\%CI: 1.22 - $5.11 \mathrm{P}=0.012$, adequate household income (AOR 2.27 95\%CI: $1.18-4.39 P=0.014$ ), first action being; taking the child to the health facility (AOR 2.45 95\%CI: $1.26-4.76 \mathrm{P}=0.008$ and having health education (IEC) done in the community (AOR 2.14 95\%CI: $1.10-4.13 P=0.024$ ). Non availability of antimalarial drugs at health facilities and self-treatments with antipyretics or herbal medication were reported in FGDs to be associated with delays in seeking appropriate malaria treatment.

Conclusion: The findings of this study highlight the barrier that exits in accessing prompt malaria treatment in a rural setting of Zambia. It underscores the need to formulate and implement interventions aimed at fostering appropriate health seeking behaviors in caretakers of under five children through community health education and addressing socioeconomic barriers that exits in rural setting.
\end{abstract}

\section{Background}

Malaria remains a major public health problem and continues to contribute significantly to infant mortality and morbidity in Africa, despite being a treatable and preventable condition (Masiye and Rehnberg, 2005). Annually, approximately 300-500 million cases and over 1 million deaths are recorded globally in children below the age of five years. Majority of these malaria infections, occur in the sub-Saharan regions were the most complicated forms of the infection are prevalent (Teklehaimanot and Bosman, 1999; Tarimo and Mismanage, 1998). Studies done in Zambia show that, close to 40 percent of infant mortality rate, 20 percent of maternal mortality rate and 45 percent of hospital admissions and outpatient department visits, can be attributed to malaria (Tuba, 2010 and $\mathrm{MoH}, 2010$ ).

Although fever is a common presentation among childhood illnesses, malaria still accounts for the vast majority of these febrile illnesses (Tarimo et al., 2000). Prompt malaria diagnosis in all childhood febrile illnesses and appropriate treatment is critical in reducing childhood related morbidity and mortality (WHO, 2005 and Schellenberg et al., 2003). The majority of children with fever receive their initial treatment at home and outside the formal health care system (William and Jones, 2004). The Zambia Malaria Indicator Survey (ZMIS) for 2010 showed that, of the 34 percent children who had a febrile episode, only 19 percent received prompt malaria treatment. Children under the age of five years are the hardest hit by malaria 
infection because they lack the acquired immunity towards the infection. Thus, delay in seeking prompt treatment could cause the infection to progress from mild to severe forms and ultimately death within the first 24 hours of onset of symptoms (Baume, 2002).

Caregivers choice of were to seek prompt malaria treatment, is influenced by accessibility to health care, severity of presumed malaria infection and caregivers level of education (Schellenberg et al., 2003; Miguel et al., 1998 and Muller et al., 2003). In addition, other factors have been mentioned to have had a direct bearing on the treatment seeking behaviors of the caregivers namely, the cultural beliefs about the cause, treatment or prevention of malaria, drug stock outs at the health facilities and use of fever relief methods at home such as antipyretics or herbal medications (Schellenberg et al., 2003).

\section{Methods}

\section{Study area and period}

This analytical cross sectional study took place from $4^{\text {th }}$ March 2013 to $14^{\text {th }}$ March 2013 in 8 rural health centers: Mpika Urban, Chilonga Hospital affiliated center, Chalabesa, Kabinga, Kasenga, ZNS and Chibansa in Mpika district. Mpika district has a population of 261,425 with a population growth of 3.8 percent (CSO, 2010). The local economy is based on agriculture, thus most households earn a living through subsistence farming. This region was chosen for the study because of its high malaria endemicity especially during the rainy season. Malaria incidence in the district increased from 185.6/1000 to 441.7/1000 population and malaria related mortality in children under 5 years of age also increased from 48/1000 to 90/1000 from 2008 to 2010, respectively (2010 Mpika DHMT Action plan report).

\section{Study population}

The study populations were caregivers with children under five years of age diagnosed with malaria, attending the selected rural health centers.

\section{Sample size and sampling procedures}

The sample size calculation was based on assumption that the proportion of under-five children who received prompt malaria treatment was 34 percent (Zambia national malaria indictor survey, 2010). The statistical significance level of 5 percent was used with coefficient set at 1.96. Using the formulae and taking into account a non response of $10 \%$, the final sample size of 380 participants was obtained.

Multiple data collection techniques were employed to ensure validity and reliability of the results namely caregivers structured interviews $(n=380)$ and focused group discussions $(n=$ 8). For the structured interviews, a multi-stage sampling was used. The first stage was sampling of health centers. The health centers were identified through probability proportion to size sampling, from a sampling frame of all the 25 rural health centers (RHCs) in Mpika district. The second stage involved sampling of the caregivers attending the health centers with their under five children diagnosed with malaria. Study participants meeting the inclusion criteria at the selected health centers were identified through simple random sampling and were recruited daily for exit interviews until the desired sample size was achieved for that particular health center. The number of caregivers to be sampled at each rural health center was determined by the catchment population of that particular health facility proportional to the total cumulative catchment population of all the eight chosen rural health centers.

Purposive sampling was used to select participants for Focus Group Discussions (FGDs). For each facility, the venue was chosen were the FGD was conducted upon completion of the individual survey interviews. A total of eight FGDs were conducted with groups composed of $8-10$ caregivers at each health facility. 


\section{Data Analysis}

Quantitative data were double entered in an EPI DATA computer software package. Then it was checked for errors and cleaned by checking for information from questionnaires before being transferred to STATA (Version 11) for analysis. The data analysis types used were descriptive and analytical analysis. In descriptive analysis, frequency counts, graphical illustrations and cross tabulations were derived. Logistic regression analysis was used for analytical analysis. First, univariate logistic regression analyses of each independent variable with the dependent variable were performed initially. Secondly, from the univariate analysis, variables that were found to be statistically significant were then entered in the multivariate logistic regression analysis using stepwise forward and backward methods to control the effect of confounders. Adjusted odds ratios, p values and confidence intervals were computed from the best model were used to interpret the study findings. In this study, the statistical significance level of 5 percent (i.e. p value $\leq 0.05$ ) was used. The variables found not to be statistically significant in the univariate analysis were excluded in the multiple regression analysis. Textual data that was derived from Focus Group Discussions was analyzed using qualitative content analysis. The recorded data was first transcribe and followed up by cross checking of recorded data with transcripts. Then textual notes were read and reread in order to gain an understanding of their content. This was followed by classification of questions, numerical coding of themes and categorizing of themes according to the most frequent used themes from different FGDs done at the other health facilities. The qualitative data provided a much deeper insight with regard to the barriers associated with prompt malaria treatment in the district.

\section{Ethical Consideration}

Ethical approval was obtained from the University of Zambia Biomedical Research Ethics Review Committee (Ref: 008-02-13). Mpika District Community Health Office gave permission for the study to be conducted in the district. Informed consent was sought from the participants for their participation in the FGDs and in the in the semi and structured interviews. Participant's confidentiality and anonymity were preserved. Moreover, Participants were informed that they were free to withdraw from the study at any time without any repercussions.

\section{Results}

\section{Socio -demographic characteristics of participants}

A total of 380 caregivers were interviewed. The majority of study children were males aged between 13-24 months 110/380 (29.0 percent) and their primary caregivers were mostly mothers 293/380 (77.1 percent), aged between 25-34 years 154/380 (44 percent). The majority of the caregivers only went as far as primary level education (65.3 percent) while 12.1 percent had no formal education. Similarly, 234/380 (61.6 percent) of heads of household had attained primary education while 7.9 percent had no formal education. Most of the heads of households were earning their living through subsistence farming 313/380 (82.4 percent) whose household income was inadequate 268/380 (70.5 percent) (Table1).

Table 1: Socio-demographic characteristics of participants

\begin{tabular}{ll}
\hline Age of children with malaria & Percentage \% (n/N) \\
\hline $0-12$ months & $20.5 \%(78 / 380)$ \\
$13-24$ months & $29.0 \%(110 / 380)$ \\
25-36 months & $19.7 \%(75 / 380)$ \\
$37-48$ months & $11.8 \%(45 / 380)$ \\
49-59 months & $19.0 \%(72 / 380)$ \\
\hline
\end{tabular}

Gender of the children

Male

$52.4 \%(119 / 380)$ 
Age of caregiver

15-24 years

$30.8 \%(117 / 380)$

25-34 years

$40.5 \%(154 / 380)$

$35-44$ years

$21.8 \%(83 / 380)$

$>45$ years

$6.40 \%(26 / 380)$

\begin{tabular}{ll}
\hline Relationship of caregiver to the child & \\
Mother & $77.1 \%(293 / 380)$ \\
Father & $17.1 \%(65 / 380)$ \\
Grandmother & $5.0 \%(19 / 380)$ \\
Others & $0.80 \%(3 / 380)$ \\
\hline Education level of caregiver & \\
No School & $12.1 \%(46 / 380)$ \\
Primary & $65.3 \%(248 / 380)$ \\
Secondary & $21.8 \%(83 / 380)$ \\
College & $0.80 \%(3 / 380)$ \\
\hline Education level of head of household & \\
No school & $7.90 \%(30 / 380)$ \\
Primary & $61.6 \%(234 / 380)$ \\
Secondary & $27.6 \%(105 / 380)$ \\
College & $2.90 \%(11 / 380)$ \\
\hline Occupation of head of household & \\
Employed & $9.40 \%(36 / 380)$ \\
Business & $8.20 \%(31 / 380)$ \\
Farmer & $82.4 \%(313 / 380)$ \\
\hline House hold income & \\
Adequate & $29.5 \%(112 / 380)$ \\
Not adequate & $70.5 \%(268 / 380)$ \\
\hline
\end{tabular}

\section{The proportion of children that received prompt malaria treatment}

As low as 53/380 (13.9 percent) children diagnosed with malaria received prompt malaria treatment, while 86.1 percent (327/380) were treated after 24 hours or more of onset of malaria symptoms. The common reason cited for delayed treatment is the long distances required to travel to reach the health facilities, compounded with luck of financial resources (19.3 percent). With regard to malaria knowledge and treatment, 64.5 percent (245/380) mentioned fever as a sign they first saw in their child to suspect malaria illness. The other symptoms mentioned include convulsions (12.1percent), poor appetite (10 percent), and vomiting (9.8 percent). The commonest antimalarial drug prescribed to these children was Lufamenthrine Artemether (Coartem) (91.5 percent). However, the practice of self medication with antipyretics among caregivers was common 36.0 percent (137/380) (Table 2).

Table 2: Characteristics of participants with prompt or delayed malaria treatment.

\begin{tabular}{ll}
\hline Promptness of treating malaria & Percentages \\
\hline Children treated within 24 hours of onset of symptoms & $13.9 \%(53 / 380)$ \\
Children treated after 24 hours of onset of symptoms & $86.1 \%(327 / 380)$ \\
\hline Reasons for delay of treatment & \\
Didn't think of seeking care & $14.4 \%(47 / 327)$ \\
No one to take child to health facility & $6.7 \%(22 / 327)$ \\
No money & $19.3 \%(63 / 327)$ \\
Long distance to health facility & $31.2 \%(102 / 327)$ \\
Child feel sick at night & $28.4 \%(93 / 327)$ \\
\hline
\end{tabular}

Malaria symptoms the child presented with 
Convulsions

Fever

Poor appetite

Vomiting

Shivering

Diarrhea

Initial treatment given to child at home

Antipyretic

Antimalarial

Antibiotic

Herbal medication

Sponging

No treatment given
$12.1 \%(46 / 380)$

$64.5 \%(245 / 380)$

$10.0 \%(38 / 380)$

$9.80 \%(37 / 380)$

$1.80 \%$ (7/380)

$1.80 \%(7 / 380)$
$36.0 \%(137 / 380)$

$2.40 \%(9 / 380)$

$1.10 \%$ (4/380)

$0.50 \%$ (2/380)

$5.0 \%(19 / 380)$

$55.0 \%(209 / 380)$

The statistical analysis was performed using univariate and multivariate logistic regression. The Unvariate analysis found the following factors to be significantly associated with increasing the odds of the child receiving prompt malaria treatment: distance of less than five kilometer to the health facility [odds ratio (OR) 2.60, 95\% CI: $1.35-0.73, \mathrm{P}=0.00$ ], adequate household income (OR 2.67 95\% CI: $1.47-4.88, \mathrm{P}=0.00$ ), head of household being employed (OR 2.40 95\% CI: 1.06 - 5.44, P = 0.04), caregiver knowledgeable on malaria transmission (OR 2.76 95\% CI: 1.37 - 5.57, $\mathrm{P}=0.01$ ), sponging the child (OR 4.80 95\% CI: $1.54-14.94, \mathrm{P}=0.007)$, taking the child to the health facility as the first action (OR 3.47 95\% CI: $1.64-7.36, \mathrm{P}=0.001)$ and having health education IEC done in the community (OR 2.32 95\% CI: 1. 28 - 4.23, P = 0.006) Table 3.

Table 3: Univariate logistic regression analysis of predictors of prompt malaria treatment in under 5 children in Mpika district.

\begin{tabular}{|c|c|c|c|}
\hline Risk factor & Proportion & $\begin{array}{l}\text { Odds ratio (Confidence } \\
\text { Interval) }\end{array}$ & P value ${ }^{1}$ \\
\hline \multicolumn{4}{|c|}{ Initial malaria treatment } \\
\hline Antipyretic & $36 \%(137 / 380)$ & $0.50(0.25-0.99)$ & 0.05 \\
\hline Other treatments & $64 \%(243 / 380)$ & 1.00 & \\
\hline \multicolumn{4}{|c|}{ Malaria symptoms child had } \\
\hline Fever & $65 \%(245 / 380)$ & $0.25(0.11-0.53)$ & 0.00 \\
\hline Poor appetite & $10 \%(38 / 380)$ & $0.27(0.08-0.93)$ & 0.03 \\
\hline Vomiting & $9.8 \%(37 / 380)$ & $0.63(0.23-1.72)$ & 0.37 \\
\hline Diarrhea & $1.8 \%(7 / 380)$ & $0.48(0.04-3.47)$ & 0.39 \\
\hline \multicolumn{4}{|c|}{ Distance to health facility } \\
\hline$<5$ kilometers & $18 \%(70 / 380)$ & $2.60(1.35-4.99)$ & 0.00 \\
\hline$>5$ kilometers & $82 \%(310 / 380)$ & 1.00 & \\
\hline \multicolumn{4}{|l|}{ Household income } \\
\hline adequate & $29 \%(112 / 380)$ & $2.67(1.47-4.88)$ & 0.00 \\
\hline Not adequate & $71 \%(268 / 380)$ & 1.00 & \\
\hline \multicolumn{4}{|l|}{$\begin{array}{l}\text { Occupation of head of } \\
\text { household }\end{array}$} \\
\hline Employed & $9 \%(36 / 380)$ & $2.40(1.06-5.44)$ & 0.04 \\
\hline Self employed & $91 \%(344 / 380)$ & 1.00 & \\
\hline \multicolumn{4}{|l|}{ Gender of children } \\
\hline Male & $52 \%(199 / 380)$ & $1.23(0.68-2.23)$ & 0.49 \\
\hline Female & $48 \%(181 / 380)$ & 1.00 & \\
\hline \multicolumn{4}{|c|}{ Education level of caregiver } \\
\hline Basic education & $88 \%(334 / 380)$ & $0.85(0.35-2.01)$ & 0.70 \\
\hline Never attended school & $12 \%(46 / 380)$ & 1.00 & \\
\hline
\end{tabular}


However, initial antipyretic treatment given to the children at home (OR 0.50 95\% CI 0.25 $-0.99 \mathrm{P}=0.05$ ), children who presented with fever (OR $0.2595 \% \mathrm{CI}: 0.11-0.53 \mathrm{P}=0.00$ ), children who had poor appetite (OR $0.2795 \%$ CI: $0.08-0.93 \mathrm{P}=0.03$ ) and presence of physical barriers to the health facility (OR $0.5195 \%$ CI: $0.26-0.98 \mathrm{P}=0.04$ ), were found to decrease the likelihood of under five children receiving prompt malaria treatment. The analysis showed the absence of association between prompt malaria treatment and gender of the child (OR 1.23 95\% CI: $0.68-3.23 \mathrm{P}=0.49$ ), education level of caregiver (OR $0.8595 \%$ CI: $0.35-2.01 \mathrm{P}=0.70$ ) and source of antimalarial (OR 0.33 95\% CI: $0.08-1.11 \mathrm{P}=0.08$ ).

When all significant variables were entered in a multivariate analysis with stepwise elimination (Table 4), the following variables were found to significantly predict uptake of prompt malaria treatment among children under five years of age: distance of less than five kilometers to the health facility (Adjusted OR 2.45 95\%CI: $1.22-5.11 \mathrm{P}=0.012$ ). This shows that caregivers who stay less than $5 \mathrm{~km}$ from the health facility were 2.5 times more likely to have prompt malaria treatment for their children than those staying more than $5 \mathrm{~km}$ from the facility. Household income (OR 2.27 95\% CI: $1.18-4.39 \mathrm{P}=0.014$ ), caregivers whose income was said to be adequate were 2.3 times more likely to seek prompt malaria treatment, than those whose income was said to be inadequate. Furthermore, knowledge on malaria transmission (Adjusted OR $2.0495 \%$ CI: $0.97-4.28 \mathrm{P}=0.049$ ), this shows that caregivers who were knowledgeable about malaria transmission were 2 times more likely to seek prompt malaria treatment. The analysis also showed that taking the child to health facility as the first action (Adjusted OR 2.45 95\% CI: $1.26-4.76 \mathrm{P}=0.008$ had the percent change in odds for SD (standard deviation) of 47.7). This means that taking the child to the facility as the first action 1 standard deviation more, increases the odds of prompt malaria treatment by $48 \%$. Also fever as the symptom (Adjusted OR $0.3695 \%$ CI $0.19-0.69 \mathrm{P}=$ 0.36 had a percentage change in odds for SD of -38.8). This means that having fever as a symptom of malaria 1 standard deviation more decrease the odds of prompt malaria treatment by 39\%. Having health education IEC done in the community (Adjusted OR 2.14 95\% CI $1.10-4.13 \mathrm{P}=0.024$ ), increases the likelihood of prompt malaria treatment. The following variables were not significantly associated with prompt malaria treatment in under five children: initial malaria treatment with antipyretics (Adjusted OR 0.58 95\% CI: $0.28-1.19$ P $=0.138$ ), occupation of the head of household (Adjusted OR 1.31 95\% CI: $0.52-3.30 \mathrm{P}=$ 0.060) and caregiver's knowledge on malaria transmission (Adjusted OR 2.04 95\% CI 0.97 $4.28 \mathrm{P}=0.05)$. This means that, caregivers who gave their children antipyretics as initial home treatment were 0.58 more likely to seek malaria treatment late, than caregivers who gave other treatments. 
Table 4: Multivariate logistic regression analysis of predictors of prompt malaria treatment in under 5 children in Mpika district.

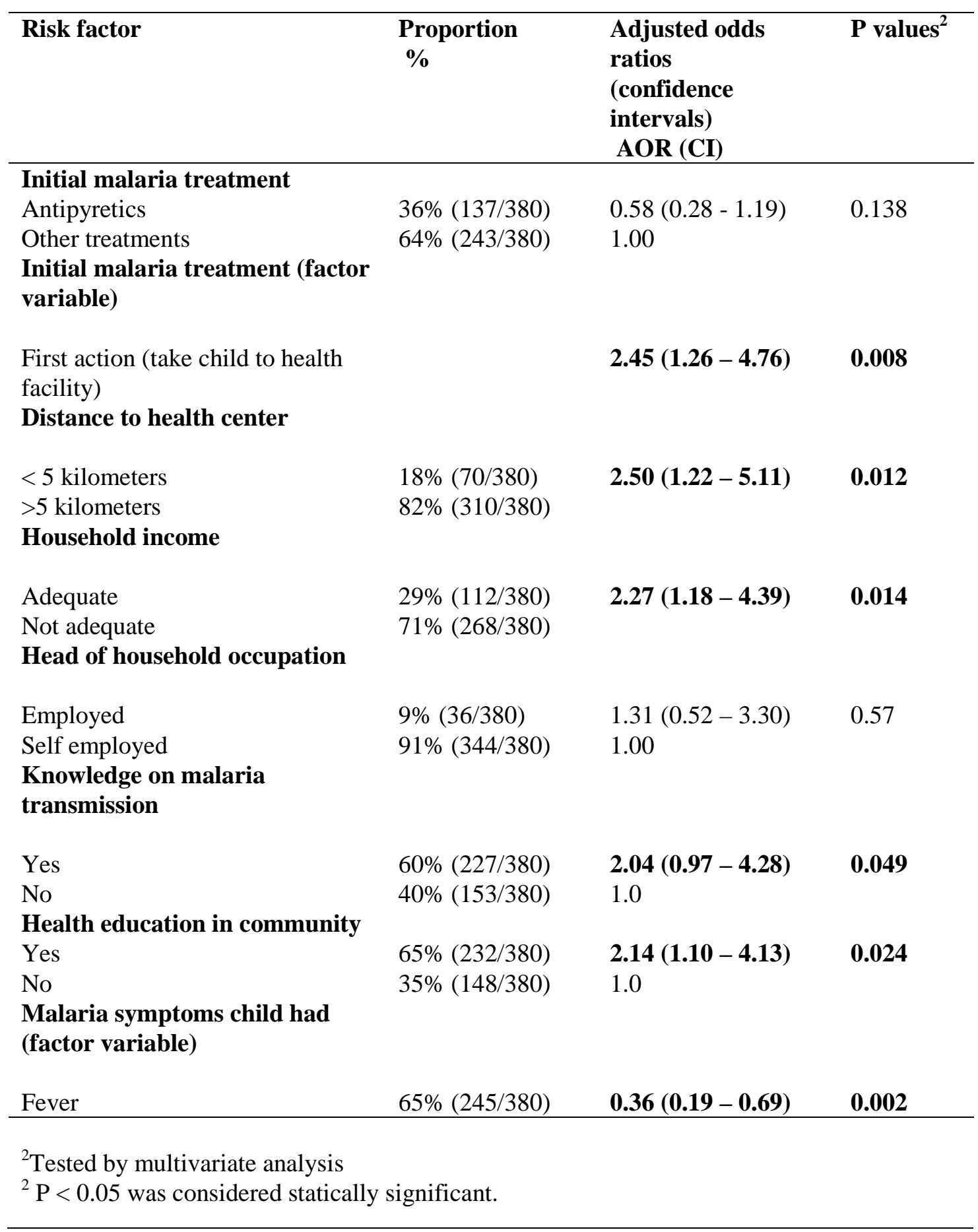

Qualitative data from FGDs highlighted the following barriers to prompt malaria treatment.

\section{Distance and transport to the health facility}

Availability of transport and physical location of the health facility has a bearing on prompt access of health services by the users. Statistical analysis revealed that children of caregivers staying more than $5 \mathrm{~km}$ from the health facility were associated with delayed malaria treatment, equally during FGDs, long distances that caregivers needed to travel either by foot or cycling to reach the health facility was a prominent theme that emerged. This was compounded by presence of physical barriers such as passing through thick forests, traversing mountains which are common physical barriers in Mpika district: 
"The problem is that we stay very far from the health center, for instance I stay in Itongo area which is very far, so if we suspect the child has malaria, we wait until the next day to see if the child will improve" (FDG, female caregiver)

\section{Perceived severity of fever}

Although malaria was recognized as a major problem in all the FGDs and fever was also positively associated as the main symptom of malaria. Caregivers however, classified fever as mild and severe body hotness. Sick children with mild body hotness were not taken to the health facility, not until their fever become severe body hotness:

"Most of the time when we see that the child has developed vomiting, shivering and mild fever, we wait for the child to develop severe body hotness also before we take the child to the health facility. Most of the time when we take such a child with mild fever, they get his blood and test, they tell us that the child has no malaria, and thus we are sent back home on some other medication. If we take the child with high fever, they always find the malaria parasite, so that's the reason we wait at home for the child to develop high fever" (FGD, male caregiver).

\section{Initial treatment of suspected malaria}

In malaria endemic areas, the use antipyretics drug to treat fever episodes is a common practice which negatively impact on prompt access to appropriate malaria treatment. In the study the common initial home treatment for suspected malaria illness, that emerged from the FGDs was the use of antipyretics such as paracetamol, which were mostly left over medication from the previous illness or procured from the local shops "tutembas". However, use of antipyretics was cited as the cause of delay in accessing care for the sick child, since the fever would normally subside after taking paracetamol, thus the caregiver could interpret this as an improvement in the child's condition:

"what causes us not to bring the child the same day they develop fever is that we give the child panadol, the fever goes, so when we wake the following day the child feels much better, so we don't bring the child to the health center" (FGD, female caregiver)

\section{Non availability of antimalarial drugs.}

The availability of drugs at a health facility is an important factor affecting access to malaria treatment. A persistent stock out of antimalarial drugs at the health centers is another barrier that emerged from the FGDs and interviews with rural health center staffs. The reasons attributed to the drug stock outs was mainly erratic RHC essential medicine and supplies of kits from the District health office and huge malaria burden in the catchment populations.

"When we take our sick children at the health center, sometimes the health workers there tell us they don't have medicine to treat malaria, they refer us to either Mpika district hospital or Chilonga general hospital were such medicine are readily available, so we have to go back to the village to prepare ourselves to go to these hospitals which are very far way" (FGD, female caregiver)

"What happens is that when the community hears that the medicine kit has arrived at the health center, they all come in numbers such that within a week, the health center runs out of all the antimalarial, and RDT test kits.” (FGD, male caregiver)

\section{Use of herbal medication}

The use of herbal medicine is imbedded in the cultural belief that herbs are effective in treating febrile convulsions (Iloeje, 1989). The FGDs revealed that, some caregivers were able to link febrile convulsions to malaria, and these mentioned that when their child with fever starts convulsing they rush the child to the health center to get treatment. However, other caregiver could not link febrile convulsions to malaria, thus associated convulsions to epilepsy. The initial treatment for febrile convulsion was giving the child tradition medicine 
mostly from advice from their parents or in laws. When the condition of the child worsened, that's the time the child is taken to the health center.

"Some of our parents or in- laws tell us to use herbal medication especially if the child is convulsing (umusanfu)" (FGD, female caregiver).

"Herbal medication was given to my child when he started fitting, but there was no improvement, until I just decided to bring the child to the hospital, that's when the child got better" (FGD, female caregiver).

\section{Discussion}

This study explored the barriers to prompt and effective malaria treatment in children aged below 5 years, in Mpika district. A wide range of interconnected factors at both household and health system level are said to influence access to early and efficacious malaria treatment in children under five years of age (Hetzel et al, 2007). The study identified the following factors to having an impact on prompt and effective malaria treatment: distances to health facilities, household income, knowledge on malaria symptoms, its transmission and treatment, community health education on malaria, availability of antimalarials at the health facilities and staffing levels at health facilities.

This study found that a low proportion of children diagnosed with malaria received prompt and effective malaria treatment in the rural district of Mpika. A similar finding was obtained from a study done in rural Blantyre- Malawi (Hetzel et al., 2008). Clearly prompt malaria treatment still remains a challenge in most rural settings, despite it being a cornerstone in prevention of malaria complications in children (Baume, 2002). The prompt use of an effective malaria treatment was equally low among study participants. Most children with malaria were initially treated with an antipyretic such as paracetamol, before being brought to the health facility. This finding is also consistent with a study done in Tanzania which indicated that as high as $78 \%$ of children received an antipyretic as first action at home prior to being taken to the health facility (Hetzel et al., 2008). Self-treatment is the most common initial treatment in childhood malaria, especially in rural areas of Africa, where three quarters of malaria cases occur ((Hetzel et al., 2008). Although antipyretics are widely used as a treatment options for childhood fevers, these are not biomedical recommended treatment for malaria. Self-treatment of malaria is a recipe for disaster as most such children are at risk of developing complicated malaria, which if not managed accordingly, is associated with a high case fatality.

The study also found that caretakers living within 5 kilometer radius of the health facilities were more likely to access prompt malaria treatment for their children. This finding is in support of the study done in Uganda which highlighted that, caretakers who traveled greater than $5 \mathrm{~km}$ to the health facilities were more likely to have delayed malaria treatment than those that travelled less than 5 kilometers to the health facilities (Rutebemberwa et al., 2009). Long distance has been pointed out by many studies as a common barrier to formal health care in rural settings (Baume, 2000). From FGDs, it was clear that long distance more often than not, make caretakers adopted a wait and see approach (Chibwana et al., 2009). Moreover, some of the strategies being advocated to combat the problem of long distance include: the use of volunteers called Community Health Workers (CHW) trained to conduct Home based management of malaria (HMM). The current HMM involves the CHW making a confirmed malaria diagnosis with Rapid diagnostic testing (RDT) then providing an appropriate antimalarial to malaria children within the community (WHO, 2005). The use of CHWs is widely practiced in many countries in Africa and some parts of Asia (WHO, 2005). Evidence that they are effective is broadly encouraging, where adequate training, supply of drugs and supervision can be maintained, but this is not a light undertaking. The study also highlighted that only a quarter of caretakers were fathers and over three quarters were mothers. Studies have shown that male involvement in the management of child illness improves access to formal health care (Halwindi et al., 2013). This can be attributed to the fact that more often than not, fathers play a pivotal role of regulating women/child access to 
prompt malaria treatment through control of home income, women mobility and health care decision (Halwindi et al., 2013).

The cost of seeking health care has often been mentioned as a major hurdle to malaria treatment in the sub-Saharan Africa (Chuma J, Okungu V, and Molyneux C, 2010). Inadequate household income was cited as one of the barriers to prompt malaria treatment in the study. This finding is consistent with the fact that household income has a bearing on access to health care services availalable to the caretaker, as those with adequate income are able to access the health facilities easily as opposed to self-medication. Mpika district is mostly rural, as such most study participants are peasant farmers with seasonal household income, this coupled with transport costs and other opportunity costs of taking children to the health facilities instead of farming, all interact to make access to effective malaria treatment more difficulty for poor households. This challenge can be addressed by implementing favorable economic policies for local peasant farmers in the district. At present, the local farmers are faced with a lot of challenges such as poor farming methods and lack of favorable markets for their farm produce. Therefore, any policy aimed at improving the livelihood of these farmers will need to be structured around such important issues. The potential spin off of such an undertaking will not only improve health service accessibility but also improve their standard of living, thus significantly reducing vulnerability to illnesses of their children.

The majority of caretakers in the study were knowledgeable on the cause, transmission, symptoms and treatment of malaria. Furthermore, caregivers who were more knowledgeable about malaria were more likely to seek prompt malaria treatment for their under 5 children than those that were not knowledgeable. However, other studies done in Africa showed that knowledge of caretakers on malaria transmission does not always translate into prompt malaria treatment in under 5 children, though such knowledge only tend to improve personal protective behaviors such as use of insecticide treated bed nets (Ahorlu, 2006 and Rutebemberwa, 2009). It is clear that, for strategies aimed at increasing caretaker's knowledge on malaria to have any meaningful impact in fostering better treatment seeking behaviors, other external factors that influence it need to be put into context.

The study demonstrated that community health education on malaria increased the likelihood of prompt malaria treatment among under five children. However, during FGDs, caretakers revealed that most of the health education sessions on malaria were conduct at the health facility by health staffs when caretakers took their children for routine under five immunization days, as opposed to being conducted in the community. There is need to broaden the target population for the health education sessions to include caretakers to do not come to health facilities. To tackle this challenge, need to increase awareness through health campaigns in the community and schools through audio-visual means such drama. Although the extent to which such social mobilization campaigns influence change in health seeking behaviors has not been exhaustively investigated, studies that have examined this issue do not consistently show a correlation between care seeking and malaria related knowledge.

The survey of health facilities revealed that most rural health centers in the district were poorly manned and experienced frequent antimalarial drug stock outs. This lead to the available limited staff being overburdened by huge patient turn over. The antimalarial stock out resulted in patients being referred to the two hospitals further away to access malaria treatment, a similar finding in a study done in Malawi (Becher et al, 2011).

In Zambia the first line malaria treatment is the use of Artemisinin-based combination treatment (ACT), which can only be obtained at the health facility with regard to most rural settings. It's worth noting that the health centers are supplied with few antimalarial in the drug kit which they received monthly from the district hospital thus resulting in non availability of medicines at the health centers. Therefore, it is important that national malaria strategies increase the allocation of ACTs in the drug kits to improve essential medicine commodity security at facility level, thus improving prompt access to effective anti-malarial treatment. The staff attrition was found to be high in the district, thus more trained health care 
provider are needed if the vision of quality health care provision is to be realized in the district.

The study had some limitations. First, the study was conducted in selected rural health centers in Mpika District and the extent to which findings can be generalized to the entire District and other settings is assumed. Second, the study participants were caregivers with sick children under five years of age, accessing care at the health facility; however those caregivers with malaria children but who did not come to the health facilities could not be interviewed introducing a form of bias. Despite these limitations, the finding from this study brings to the fore some of the factors that influence prompt malaria treatment in a rural setting and provide a platform on which future malaria control strategies can be formulated. However, there is need for further research using other methodologies to explore in depth these factors that promote or negatively impact on prompt and effective malaria, in order to have a much deeper perception of not only structural but also the cultural and socio factors at play.

\section{Conclusion}

The results from this study suggest that a low proportion of caretakers of children under five years of age with malaria are seeking care promptly at health facilities in rural settings. Barriers to access of prompt health care include longer distances of travel to health facilities, inadequate household income, shortage of antimalarial at facilities encourages caregivers to the practice of self-medication with other non-biomedically approved malaria treatments such as antipyretics or herbal medication, resulting in delayed malaria treatment. Factors that negatively impact on access to prompt malaria treatment are so complex such that even in a country, they differ from one locality to the other. Therefore there is need to look at the local context in coming up with solutions. Finally, there is an urgent need to direct additional resources towards addressing some of the barriers identified in the study, to reduce the morbidity and mortality associated with malaria among the under five children in the rural setting in order for the country to attain Sustainable Development Goals of reducing malaria infections and infant mortality rates in Zambia by 2030.

\section{Acknowledgements}

The author thanks Mpika District Medical Community Health Office, for allowing the study to be conducted in the district. The health center staffs and caregiver of under five children for providing much needed information. I also thank my family and friends for support and encouragements during the study. The author declares that there are no competing interests.

\section{References}

[1] Ahorlu C K, Koram K A, Ahorlu C, De Savigny D \& Weiss M G (2006) Socio-cultural determinants of treatment delay for childhood malaria in southern Ghana. Tropical Medicine and International Health 11, 1022-1031.

[2] Becher H, Muller O, Jahn A, Gbangou A, Kynast-Wolf G \& Kouyate B (2004) Risk factors of infant and child mortality in rural Burkina Faso. Bulletin of the World Health Organization 82, 265273.

[3] Baume, C. (2002). A Guide to research on care-seeking for childhood malaria. Arlington, Virginia: Sara, Basics ii. 46(30): 256-59.

[4] Central statistical office Zambia (CSO, 2010).

[5] Chibwana AI, Mathanga DP, Chinkhumba J, Campbell CH (2009) Socio-cultural predictors of health-seeking behaviour for febrile under-five children in Mwanza-Neno district, Malawi. Malaria Journal 8: 219.

[6] Chuma J, Okungu V, Molyneux C. (2010) Barriers to prompt and effective malaria treatment among the poorest population in Kenya. Malar J 9: 144. 
[7] Halwindi, H, S. Siziya, P. Magnussen, and A. Olsen (2013). Factors Perceived by Caretakers as Barriers to Health Care for Under-Five Children in Mazabuka District, Zambia. ISRN Tropical Medicine. 10.1155

[8] Hetzel M, Iteba N, Makemba A, Mshana C, Lengeler C, et al. (2007) Understanding and improving access to prompt and effective malaria treatment and care in rural Tanzania: The ACCESS Programme. Malar J 6: 83.

[9] Hetzel M W, Obrist B, Lengeler C, Msechu J J, Nathan R, et al. (2008) Obstacles to prompt and effective malaria treatment lead to low community-coverage in two rural districts of Tanzania. BMC

Public Health. 8: 317. [PMC free article] [PubMed

[10] McCombie SC. (2002) Self-treatment for malaria: the evidence and methodological issues. Health Policy Plan.17:333-344. doi: 10.1093/heapol/17.4.333. [PubMed]

[11] Miguel, C.A., Manderson, L., \& Lansang, M.A. (1998). Patterns of treatment for malaria in Tayabas, the Philippines; implications for control. Trop Med Int Health. 3:413-421

[12] Ministry of Health (MoH). Actions for Scale-up for Impact on Malaria in Zambia. National malaria strategic plan 2006 - 2010.

[13] Muller O, Traore C, Becher H, Kouyate B. (2003) Malaria morbidity, treatment seeking behaviour, and mortality in a cohort of young children in rural Burkina Faso. Trop Med lnt Health. 8(4):290-296. PubMed Abstract

[14] Rutebemberwa E, Kallander K, Tomson G, Peterson S \& Pariyo G (2009) Determinants of delay in care-seeking for febrile children in eastern Uganda. Tropical Medicine and International Health 14, 472-479.

[15] Schellenberg J. R. M. A., Victora C. G., Mushi A., De Savigny D., Schellenberg D., Mshinda H., Bryce J. (2003) Inequities among the Very Poor: Health Care Children in Rural Southern Tanzania. Lancet. 361:561-66. [PubMed]

[16] Tarimo D S, Lwihula G K, Minjas J N, Bygbjerg I C. (2000) Mother's perceptions and knowledge on childhood malaria in the holoendemic Kibaha district, Tanzania: implications for malaria control and the IMCI strategy. Trop Med Int Health. 5:179-84.

[17] Tarimo, D. S., Urassa, D. P., Msamanga, G. I. (1998). Caretakers' perceptions of clinical manifestations of childhood malaria in holo-endemic rural communities in Tanzania. East African Medical Journal. 5(3), 93-96.

[18] Teklehaimanot A, Bosman A. (1999) Opportunities, problems and prospects for malaria control in sub-Saharan Africa. Parassitologia. 41:335-338. [PubMed]

[19] Tuba M, Sandoy I F, Bloch P, Byskov J. (2010) Fairness and legitimacy of decisions during delivery of malaria services and ITN interventions in Zambia. Malaria J. 9:309. doi: 10.1186/14752875-9-309.

[20] Mpika DHMT Action Plan 2010.

[21] Masiye, F. \& Rehnberg, C. (2005). The economic value of an improved malaria treatment programme in Zambia: results from a contingent valuation survey. Malaria Journal. 4:60

[22] Ministry of health bulletin (MoH 2010).

[23] Williams HA, Jones CO. (2004) A critical review of behavioral issues related to malaria control in sub-Saharan Africa: what contributions have social scientists made? Soc Sci Med. 59:501-23. PubMed Abstract | Publisher Full Text

[24] WHO/UNICEF: The Africa Malaria Report 2003. World Health Organization/UNICEF, Geneva; 2003.

[25] WHO: The Roll Back Malaria strategy for improving access to treatment through home management of malaria. Geneva, World Health Organization (WHO/HTM/MAL/2005.1101); 2005.

[26] Zambia malaria indicator survey, (ZMIS 2010) 